\title{
Maintaining Diversity in Agent-Based Evolutionary Computation
}

\author{
Rafał Dreżewski and Marek Kisiel-Dorohinicki \\ Department of Computer Science, \\ AGH University of Science and Technology, Kraków, Poland \\ \{drezew, doroh\}aagh.edu.pl
}

\begin{abstract}
Niching techniques for evolutionary algorithms are aimed at maintaining the diversity through forming subpopulations (species) in multi-modal domains. Similar techniques may be applied to evolutionary multi-agent systems, which provide a decentralised model of evolution. In this paper a specific EMAS realisation is presented, in which the new species formation occurs as a result of co-evolutionary interactions between preexisting species. Experimental results aim at comparing the approach with a classical niching techniques and a basic EMAS implementation.
\end{abstract}

\section{Introduction}

The term Evolutionary Computation is usually used to describe a wide range of global search and optimization techniques based on analogies to natural evolutionary processes. However, both experiments and analysis show that for multi-modal problem landscapes a simple evolutionary algorithm will inevitably loose the diversity of its population and in consequence locate only a single solution-which is often a local optimum. In order to overcome this limitation some mechanism that creates and maintains different subpopulations (species) in a multi-modal domain must be used.

Traditionally, there are two basic approaches in evolutionary biology to understanding speciation process [3]. The first one called allopatric speciation occurs when subpopulations of a given species become geographically isolated. After isolation they follow different paths of evolution, which eventually lead to forming of different species. The second kind of speciation is called sympatric speciation. Such speciation results from niche separation due to resource competition, predator-prey co-evolution, sexual selection, etc. In this case speciation process takes place within single population.

Evolutionary multi-agent systems assume a decentralised model of evolution [5], which incorporates mechanisms of both allopatric (geographical isolation due to physical distribution of subpopulations) and sympatric (competition for limited resources in energy-based selection) speciation. In fact, these mechanisms prove not powerful enough to maintain stable subpopulations locating different optima. That is why the idea of co-evolutionary multi-agent system (CoEMAS) was introduced [2], which opens new possibilities of modeling biological speciation mechanisms based on co-evolutionary interactions-like predator-prey interactions, sexual preferences, etc. 


\section{Maintaining Diversity in Evolutionary Algorithms}

During the years of research various mechanisms and techniques for creating and maintaining species located within the basins of attraction of local minima (niches) have been proposed. All these niching techniques allow species formation via the modification of the parent selection mechanism (fitness sharing [4]), the modification of mechanism of selecting individuals for new generation (crowding [6]), the restriction of application of the selection and/or recombination mechanisms (by grouping individuals into subpopulations or by introducing the environment with some topography in which the individuals are located [1]).

In co-evolutionary algorithms the fitness of each individual depends not only on the quality of solution to the given problem but also (or solely) on other individuals' fitness. This makes such techniques applicable in the cases where the fitness function formulation is difficult (or even impossible). Co-evolution of species was also used as a mechanism for maintaining population diversity, introducing open-ended evolution and improving adaptive capabilities of evolutionary algorithms in dynamic environments. As the result of ongoing research quite many co-evolutionary techniques have been proposed. Generally, each of these techniques belongs to one of two classes: competitive [7] or cooperative [8].

\section{Evolutionary and Co-evolutionary Multi-agent Systems}

The main idea of evolutionary multi-agent system is the modeling of evolution process in MAS [5]. In opposition to classical evolutionary algorithms, in EMAS there is no centralized algorithm which manipulates the whole population. All individuals (agents) are independent and make their own decisions, particularly these concerning reproduction and death.

Selection in EMAS is based on a non-renewable resources, which are possessed by the agents. Every activity costs some resource and may be realized provided that the agent's resource level is high enough. Resources can be gained only from the environment or other agents, and the rule is that better fit agents are given more resources than less fit ones. This means the competition for limited resources in common environment, which allows for sympatric speciation.

Agents live within an environment with a defined spatial structure and may change their location within the environment. It is thus a natural model of allopatric speciation because evolutionary processes may run with different conditions in many locations coexisting in parallel. Agents migrate, so the genetic material (information) is exchanged between locations. This model is, to some extent, similar to island model of PEA with all its advantages and disadvantages.

The key idea that differentiates co-evolutionary multi-agent system (CoEMAS) from EMAS is the co-evolution of several species (which cooperate or compete) in one environment [2]. In the particular model of EMAS with co-evolving species (NCoEMAS) there is a hierarchical structure of species: agents-solutions live within agents-niches.

In NCoEMAS there is a closed circulation of resources. Some resource is given from the environment to the agents-niches (proportionally to their fitness) and then 

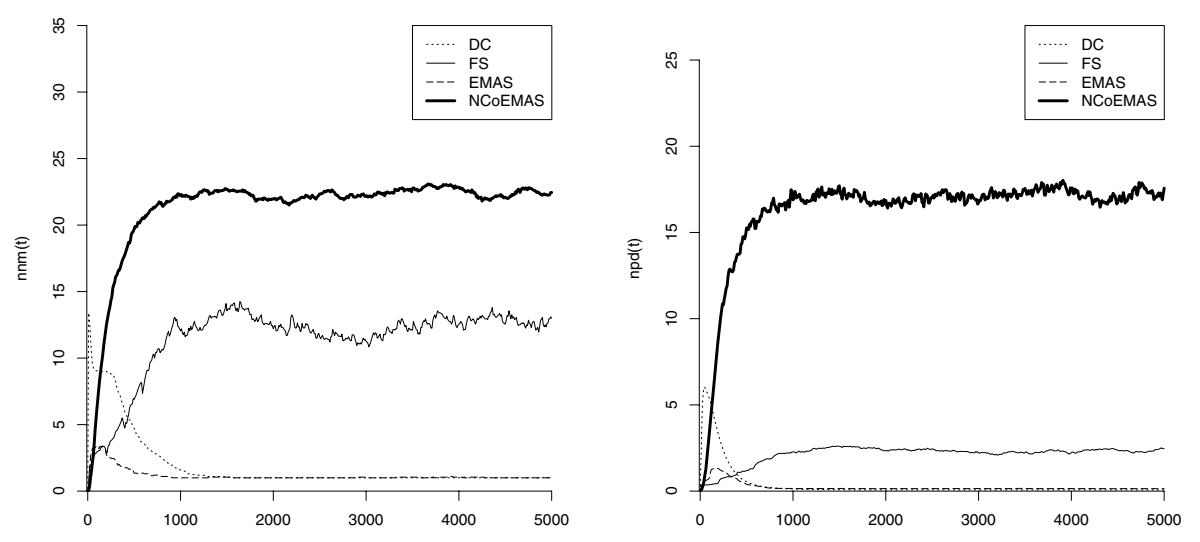

a)

b)

Fig. 1. The number of Rastrigin's function local minima neighborhoods located (a) and the value of proportional species' sizes indicator (b) - average values from 20 runs of different algorithms

redistributed among agents-solutions (a competition for limited resources). In spite of the proportional amount of resource, all agents-niches are also given some minimal amount of resource in order to keep alive less fitted species of solutions. The resource spent by the agents returns to the environment.

Each time step every agent-solution searches for the agent-niche, which is located within the basin of attraction of the same local minimum. If there are no such agentsniches, the agent-solution creates a new agent-niche, which genotype is the copy of its own genotype and migrates into it. Then each agent-solution, which have enough resource, searches its agent-niche for the reproduction partner.

In the considered case genotypes of all agents are real-valued vectors. Intermediate recombination and mutation with self-adaptation are used for agents-solutions and special mutation for agents-niches (the resulting genotype is the center of gravity of agentssolutions that belong to the agent-niche which genotype is mutated). Only agents-niches can migrate within the environment. They can also merge if they are located at the same place of environment and if they are located within the basin of attraction of the same local minimum.

\section{Experimental Results}

The experiments were carried out for four techniques: EMAS, and NCoEMAS, as well as deterministic crowding ( $D C[\overline{6}])$ and fitness sharing ( FS [4]). Figure 1] shows the average number of Rastrigin's function local minima neighborhoods located by the compared systems. The local minima neighborhood was classified as "located" when there was at least 3 individuals closer than 0.05 to that local minima. Figure $1 \mathrm{~b}$ shows the average values of proportional species' sizes indicator $n p d(t)$. The $n p d(t)$ indicator gives the higher measure when the sizes of species located within basins of attractions of local minima are proportional to the "quality" of that local minima. 
The results of experiments indicate that NCoEMAS located more neighborhoods of local minima and maintained subpopulations more stably than two classical niching techniques (fitness sharing and deterministic crowding), as well as a classical EMAS. Tendency to maintain high diversity within species was observed for $F S$, but in this case there were also agents located outside the basins of attraction of local minima. $D C$ has the strong tendency to lose located basins of attraction of local minima during the evolution process. EMAS cannot be applied to multi-modal function optimization without introducing special mechanisms such as co-evolution. It turned out that competition for limited resources and environment with a defined spatial structure are not enough to form and maintain more than one species in the case of multi-modal problems.

\section{Concluding Remarks}

Most of classical niching techniques indirectly model resource sharing within the niches. On the other hand, parallel evolutionary algorithms model speciation caused by geographical isolation of subpopulations. The approach of co-evolutionary multiagent systems combines all these techniques in one coherent model. It allows for natural implementation of the process of sympatric speciation based on niche separation due to resource competition. At the same time allopatric speciation is achieved based on environmental structure of EMAS.

The presented results of simulation experiments are promising and encourage further work on the idea of co-evolution in EMAS. It demands more experimental studies to find out which parametrs of the introduced techniques are of vast importance for their efficiency, and how the behaviur of the system changes for different kinds of problems.

\section{References}

1. E. Cantú-Paz. A survey of parallel genetic algorithms. Calculateurs Paralleles, Reseaux et Systems Repartis, 10(2):141-171, 1998.

2. R. Dreżewski. A co-evolutionary multi-agent system for multi-modal function optimization. In M. Bubak, G. D. van Albada, P. M. A. Sloot, and J. J. Dongarra, editors, Proc. of the 4th Intl. Conf. on Computational Science (ICCS 2004), LNCS, Springer-Verlag, 2004.

3. S. Gavrilets. Models of speciation: what have we learned in 40 years? Evolution, 57(10):21972215, 2003.

4. D. E. Goldberg and J. Richardson. Genetic algorithms with sharing for multimodal function optimization. In J. J. Grefenstette, editor, Proceedings of the 2nd International Conference on Genetic Algorithms, pages 41-49. Lawrence Erlbaum Associates, 1987.

5. M. Kisiel-Dorohinicki. Agent-oriented model of simulated evolution. In W. I. Grosky and F. Plasil, editors, SofSem 2002: Theory and Practice of Informatics, LNCS. Springer-Verlag, 2002.

6. S. W. Mahfoud. Crowding and preselection revisited. In R. Männer and B. Manderick, editors, Parallel Problem Solving from Nature - PPSN-II, Elsevier, 1992.

7. J. Paredis. Coevolutionary computation. Artificial Life, 2(4):355-375, 1995.

8. M. A. Potter and K. A. De Jong. Cooperative coevolution: An architecture for evolving coadapted subcomponents. Evolutionary Computation, 8(1):1-29, 2000. 